\title{
Optimal Power Management of a DISCO with Integrations of Reliability Considerations and Wind Farm Based on Benders Decomposition
}

\author{
M. Baghdadi, S. S. Mortazavi, and A. Saidian \\ Electrical Engineering Department, Shahid Chamran University, Ahwaz 61357831351, Iran \\ Correspondence should be addressed to S.S. Mortazavi, mortazavi_s@scu.ac.ir
}

Received 7 August 2010; Revised 19 April 2011; Accepted 13 June 2011

Academic Editor: Ekaterina Pavlovskaia

Copyright (c) 2011 M. Baghdadi et al. This is an open access article distributed under the Creative Commons Attribution License, which permits unrestricted use, distribution, and reproduction in any medium, provided the original work is properly cited.

This paper presents a comprehensive framework model of a distribution company with security and reliability considerations. A probabilistic wind farm, which is a renewable energy resource, is modeled in this work. The requirement energy of distribution company can be either provided by distribution company's own distributed generations or purchased from power market. Two reliability indices as well as DC load flow equations are also considered in order to satisfy reliability and security constraints, respectively. Since allocating proper spinning reserve improves reliability level, the amount of spinning reserve will be calculated iteratively. In this work, all equations are expressed in a linear fashion in which unit commitment formulation depends on binary variables associated with only on/off of units. The benders decomposition method is used to solve securitybased unit commitment.

\section{Introduction}

In general, electrical energy sector has been affected by two main factors over the past two decades. The first factor is the advancement in generation technologies which has been evolving on a continuous basis. Newer and different energy transformation resources have been introduced to achieve high standards of energy provision. The second factor is the trend to liberate the energy sector from a monopolistic operating regime to a deregulated one to establish competitive markets for electricity [1].

Traditionally, a Distribution Company (DISCO) purchases energy from wholesale market, at a high voltage level, and then transfers this energy to final customers. Nevertheless, the restructuring process of the energy sector has stimulated the introduction of new agents and products, and the unbundling of traditional DISCO into technical and commercial tasks, including the provision of ancillary services [2]. 
A day-ahead energy acquisition model for a DISCO in a pool market in the presence of financial bilateral contracts is presented in [3]. Both investor and utility-owned DG units as well as interruptible load (IL) options are considered in the model. An optimal power flow $(\mathrm{OPF})$ model is used to arrive at the optimal set of energy schedules and decisions.

A multiperiod energy acquisition model for a DISCO with DG and IL options has been presented in [4]. A bi-level optimization formulation is developed wherein the upper subproblem (SP) maximizes the DISCO's revenue, while the lower subproblem addresses the independent system operator's (ISO's) market clearing by minimizing generation costs and compensation costs for IL. The model takes intertemporal effects such as ramping into consideration.

In [5], a quantification of benefits from customer-owned back-up generators to DISCOs is carried out. An integration scheme for DGs in a pool-based market structure is proposed in [6] that encompasses both energy and capacity payment procedures. The problem of dispatch and control of DGs is formulated in [7] as a multi-agent system-based scheme, specifically for the purpose of voltage support.

Power system operators must maintain a certain amount of spinning reserve (SR) at all time for the purpose of protection in the event of a sudden loss of generating capacity or system load fluctuation. Over the last four decades, numerous techniques and methods have been developed to calculate reserve-constrained unit commitment $[8,9]$. Both deterministic and probabilistic approaches can and are used to establish spinning reserve requirements. Some utilities have adopted deterministic criteria for the spinning reserve requirements. Their operating rules require the spinning reserve to the greater than the capacity of the largest online generator or a fraction of the load, or equal to some function of both of them [10]. Reference [11] presents a probabilistic technique to determine the required spinning reserve in a traditional power system using loss of load probability (LOLP) index. A probabilistic pool-based market clearing algorithm is presented in [12] which is based on the reliability of the unit instead of the price of energy and reserve.

As wind is a variable source of energy and cannot be predicted with high accuracy. Capacity factor of wind farms compare to conventional power plants is lower. In [13], a reliability-based unit commitment in restructured power systems with high penetration of wind farms is proposed. However the output of wind farm in [13] is the same in all hours.

In this paper, a reliability-based unit commitment in a distribution company is proposed. The probabilistic output of Siahpoosh [14] wind farm is calculated in a 24 hour period. The linearized unit commitment's equations are divided into two stages and are solved with Benders decomposition method. Since this method decomposes the problem into two subproblems, the problem is going to it is widely used to solve unit commitment problem [15]. In an iterative process, reliability indices, LOLP as well as expected energy not served (EENS), will be calculated. Since in this paper the reliability unit commitment is solved in a sequential procedure, the authors add a penalty term to total unit commitment cost and put aside the first five results. The amount of spinning reserve is set in a way to satisfy adequate reliability level.

The rest of this paper organizes as follows. Section 2 describes a modeling and mathematical formulation of a distribution company. Section 3 expresses the solution. Section 4 discusses about the result of applying the proposed method on an eight test system. In Section 5, conclusion of the study is represented. A review of Benders Decomposition procedure is proposed in the appendix. 


\section{Modeling and Mathematical Formulation of a Distribution Company}

A model of the operation of DISCO in a day-ahead electricity market is depicted in Figure 1. This model consists of several parts that are described in details as follows.

\subsection{Objective Function}

As it is stated in (2.1), the objective function consists of two components. The first component expresses the cost function of disco's DG units. The real wind farm is also considered in this model as a renewable source of energy. Since the price of operation and maintenance of wind farm is very low, the cost of wind farm is set to zero. The second component considers the cost function of purchased power from the market at the day-ahead market.

According to (2.2), the total cost function consists of three elements as it is described as follows:

$$
\mathrm{OC}=\min \sum_{k=1}^{K}\left[\sum_{i=1}^{I}\left[C_{i}(k)+\mathrm{CS}_{i}(k)+\mathrm{CD}_{i}(k)\right]+C\left(P^{\mathrm{WH}}(k)\right)\right] \text {. }
$$

The first component is the operation cost that is the result of unit commitment. The second and third components are related to cost of spinning reserve and reliability, respectively. Solving the unit commitment, spinning reserve, and reliability criteria will be calculated iteratively,

$$
C_{\mathrm{TC}}=\mathrm{OC}+\sum_{k=1}^{K} C_{\mathrm{SR}}(k)+C_{\mathrm{EIC}}
$$

\subsubsection{Linerazied Operation Cost of DISCO-Owned DG}

The I/O characteristic curve of a DG is a quadratic and can be obtained from the following equation [16]:

$$
C_{i}(k)=a_{i} \cdot\left(P_{i}(k)\right)^{2}+b_{i} \cdot P_{i}^{\mathrm{DG}}(k)+c_{i}, \quad \forall i \in I, \forall k \in K .
$$

As aforementioned above, all equations should be expressed in a linear fashion. Maintaining the convexity, (2.3) can be converted to multiple piecewise linear segments in order to approximate the nonlinearity. The piecewise linear cost curve of $i$ th DG is illustrated 


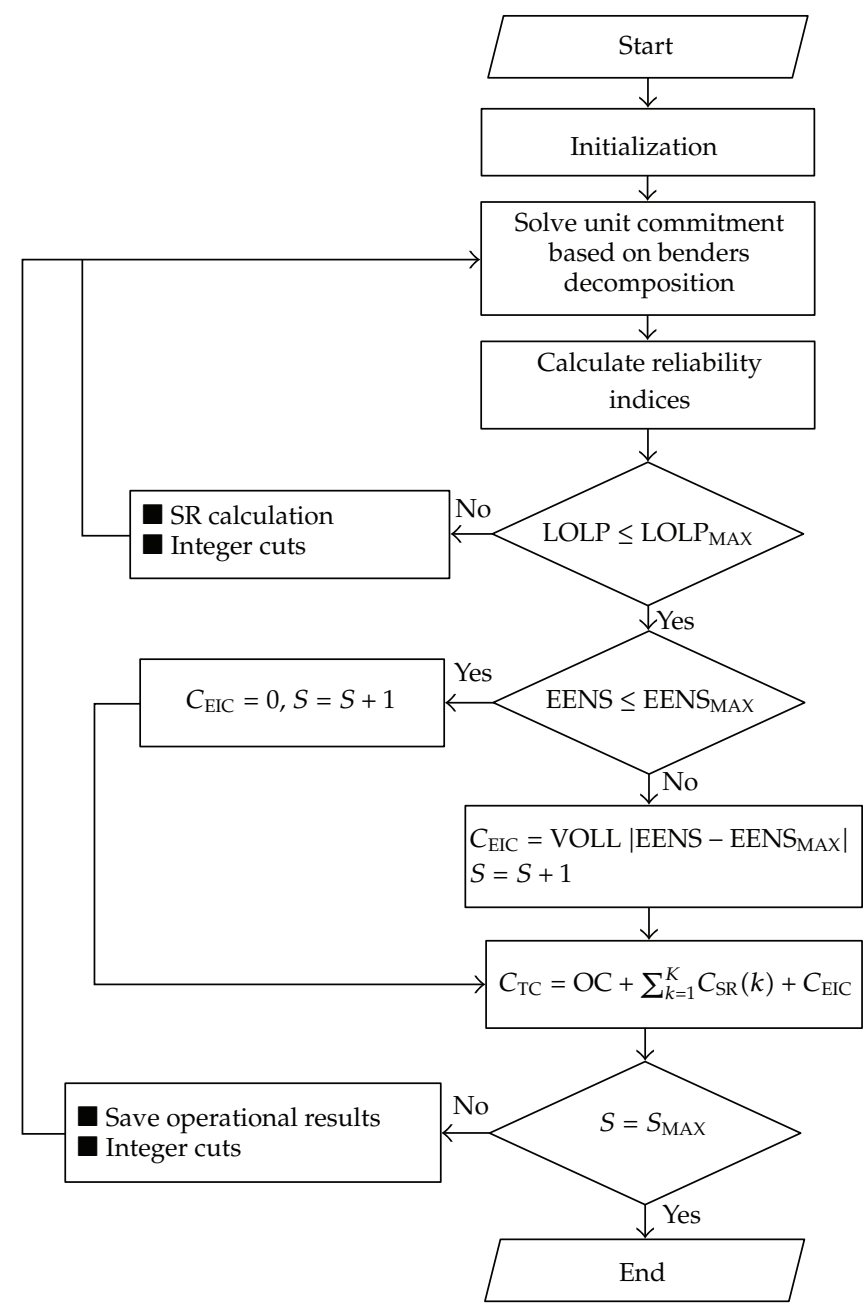

Figure 1: The Proposed Model of DISCO with reliability and security constraints.

in Figure 2. The analytic representation of this linear approximation by considering this fact that marginal costs are increasing is as follows:

$$
\begin{gathered}
C_{i}(k)=\left(a_{i}+b_{i} \underline{P}_{i}(k)+c_{i} \underline{P}_{i}^{2}(k)\right) v_{i}(k)+\sum_{l=1}^{\mathrm{NL}} F_{l, i} \delta_{l, i}(k), \quad \forall i \in I, \quad \forall k \in K, \\
P_{i}(k)=\underline{P}_{i}(k) v(k)+\sum_{i=1}^{\mathrm{NL}} \delta_{j, i}(k), \quad \forall i \in I, \quad \forall k \in K, \\
\delta_{1, i}(k) \leq T_{1, i}(k)-\underline{P}_{i}(k), \quad \forall i \in I, \quad \forall k \in K, \\
\delta_{l, i}(K) \leq T_{l, i}-T_{l-1, i}, \quad \forall i \in I, \quad \forall k \in K, \quad \forall l \in 2, \ldots, \mathrm{NL}_{i}-1, \\
\delta_{\mathrm{NL}, i}(k) \leq \bar{P}-T_{\mathrm{NL}-1, i}, \quad \forall i \in I, \quad \forall k \in K, \\
\delta_{l, i}(k) \geq 0, \quad \forall i \in I, \forall l \in 1, \ldots, \mathrm{NL}_{i}, \quad \forall k \in K .
\end{gathered}
$$




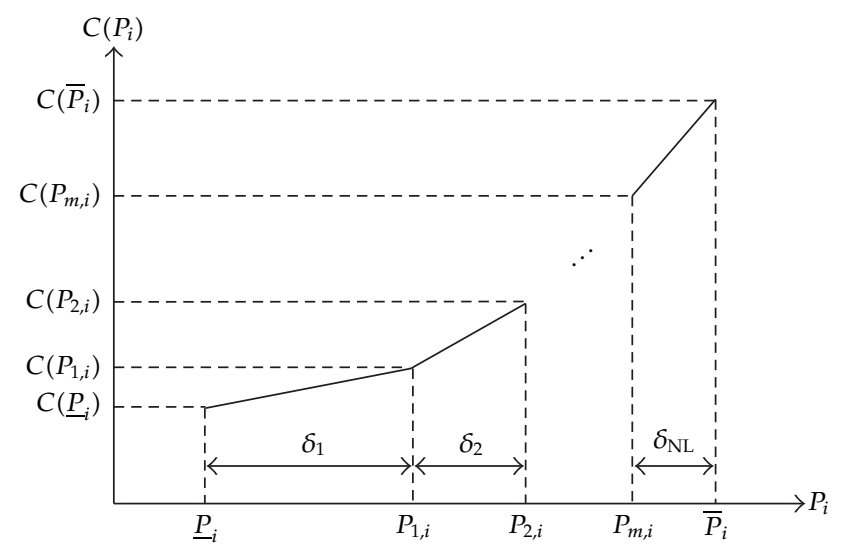

Figure 2: Piecewise linear cost curve of $i$ th DG.

\subsubsection{Power Purchased from Power Market}

The DISCO is able to obtain its needed energy from wholesalers in power market (PM):

$$
\begin{aligned}
& C\left(P^{\mathrm{WH}}(k)\right)=\rho^{\mathrm{WH}}(k) P^{\mathrm{WH}}(k), \quad \forall k \in K, \\
& P_{\mathrm{Min}}^{\mathrm{WH}}(k) \leq P^{\mathrm{WH}}(k) \leq P_{\mathrm{Max}}^{\mathrm{WH}}(k), \quad \forall k \in K .
\end{aligned}
$$

\subsection{Model Constraints}

\subsubsection{Generation Limits and Ramping Constraints}

The generation limits of unit $i$ for each period are set as follow:

$$
\begin{gathered}
\underline{P}_{i}(k) v_{i}(k) \leq p_{i}(k) \leq \bar{p}_{i}(k), \quad \forall i \in I, \quad \forall k \in K, \\
0 \leq \bar{p}_{i}(k) \leq \bar{P}_{i}(k) v_{i}(k), \quad \forall i \in I, \quad \forall k \in K .
\end{gathered}
$$

Constraints (2.6) bounds the generation by the minimum power output and the maximum available power output of $i$ th unit in period $k, \bar{p}_{i}(k)$ which is a nonnegative variable bounded by (13), the unit capacity. It should be noted that while unit $i$ is offline in period, $k, v_{i}(k)=0$. So $p_{i}(k)$ and $\bar{p}_{i}(k)$ are equal to zero.

Variable $\bar{p}_{i}^{\mathrm{DG}}(k)$ should be limited by ramp-up (or startup ramp-up) (2.7) as well as shutdown ramp rates constraints (2.8):

$$
\begin{aligned}
p_{i}(k) \leq & p_{j}(k-1)+\bar{P}_{i}\left[1-v_{i}(k)\right] \\
& +\mathrm{SU}_{i}\left[v_{i}(k)-v_{i}(k-1)\right]+\mathrm{RU}_{i} \cdot v_{i}(k-1), \quad \forall i \in I, \forall k \in K, \\
\bar{p}_{i}(k) \leq & \bar{P}_{i} v_{i}(k+1)+\mathrm{SD}_{i}\left[v_{i}(k)-v_{i}(k+1)\right] \\
& +\mathrm{RU}_{i} \cdot v_{i}(k-1), \quad \forall i \in I, \forall k \in 1 \cdots K-1 .
\end{aligned}
$$




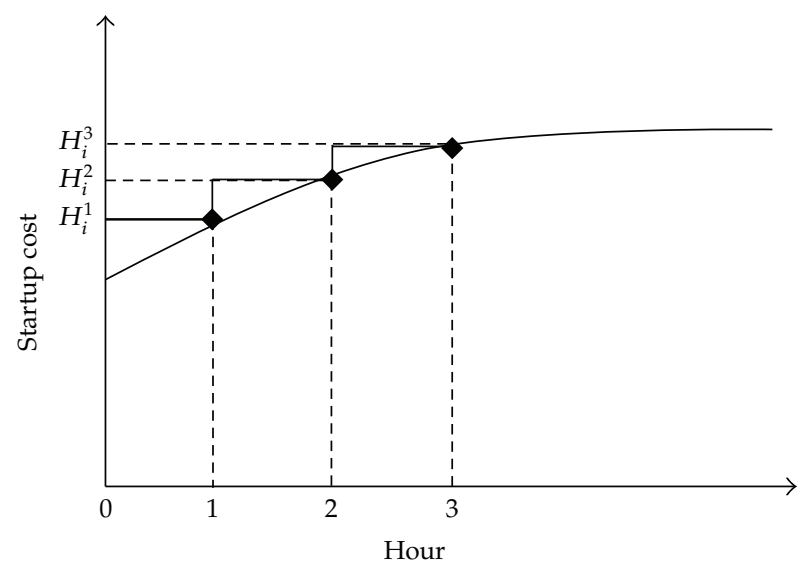

Figure 3: Exponential, discrete, and stairwise startup cost functions.

Hence, ramp-down limits are imposed on the power output

$$
\begin{aligned}
p_{i}(k-1)-p_{i}(k) \leq & \mathrm{SD}_{i}\left[v_{i}(k-1)-v_{i}(k)\right] \\
& +\bar{P}_{i}\left[1-v_{i}(k-1)\right]+\operatorname{RD}_{i}(k), \quad \forall i \in I, \forall k \in K .
\end{aligned}
$$

It should be noted that constraints (2.6) and (2.9) only include binary variables, $v_{i}(k)$, and there is no need for extra variables as used in [17].

\subsubsection{Startup and Shutdown Constraints}

As depicted in Figure 3 [18], the startup cost function of a generator has an exponential nature that can be approximated in a discrete form as a stair-wise function. A mixed-integer linear formulation for stair-wise startup cost [19] was proposed in (2.10):

$$
\begin{gathered}
\mathrm{CS}_{i}(k)=K_{i}^{t}\left[v_{i}(k)-\sum_{n=1}^{t} v_{i}(k-n)\right], \quad \forall i \in I, \forall k \in K, \forall t \in 1 \cdots \mathrm{ND}_{i}, \\
c_{i}^{u}(k) \geq 0, \quad \forall i \in I, \quad \forall k \in K .
\end{gathered}
$$

As it is clear in (2.10), the linear startup cost functions only rely on the binary variables of committed units. Moreover, considering the fuel cost, the shutdown cost while the unit is going to be offline [19], is stated as follows:

$$
\begin{aligned}
\mathrm{CD}_{i}(k) \geq & C_{i}\left[v_{i}(k-1)-v_{i}(k)\right], \quad \forall i \in I, \quad \forall k \in K, \\
& \mathrm{CD}_{i}(k) \geq 0, \quad \forall i \in I, \quad \forall k \in K .
\end{aligned}
$$




\subsubsection{Linear Expression of Minimum up- and downtime Constraints}

As it is stated in [14], minimum up- and downtime of each DG relies only on committed units, $v_{i}(k)$. Constraints regarding the minimum up time are formulated as follows:

$$
\begin{gathered}
\sum_{k=1}^{G_{i}}\left[1-v_{i}(k)\right]=0, \quad \forall i \in I, \\
\sum_{n=k}^{n=k+\mathrm{UT}_{i}-1} v_{i}(n) \geq \mathrm{UT}_{i}\left[v_{i}(k)-v_{i}(k-1)\right], \quad \forall i \in I, \quad \forall k \in G_{i}+1 \cdots K-\mathrm{UT}_{i}+1, \\
\sum_{n=k}^{T}\left\{v_{i}(n)-\left[v_{i}(k)-v_{i}(k-1)\right]\right\} \geq 0, \quad \forall i \in I, \quad \forall k \in K-U T_{i}+2 \cdots K,
\end{gathered}
$$

where $G_{i}$ is the number of initial periods during which $i$ th unit must be online. $G_{i}$ is mathematically expressed as $G_{i}=\min \left\{K,\left[\mathrm{UT}_{i}-\mathrm{UT}_{i}^{0}\right] \cdot V_{i}(0)\right\}$.

Constraint (2.12) are related to the initial status of the units as defined by $G_{i}$. Constraints (2.13) are used for subsequent periods to satisfy minimum uptime constraint during all the possible sets of the consecutive periods of size UT U $_{i}$ Constraints (2.14) model the final $\mathrm{UT}_{i}-1$ periods in which if unit $i$ is started up, it remains online until the end of the time span.

Analogously, minimum downtime constraints are formulated as follows (2.15):

$$
\begin{gathered}
\sum_{k=1}^{L_{i}} v_{i}(k)=0, \quad \forall i \in I, \\
\sum_{n=k}^{n=k+\mathrm{DT}_{i}-1}\left[1-v_{i}(n)\right] \geq \mathrm{DT}_{i}\left[v_{i}(k-1)-v_{i}(k)\right], \quad \forall i \in I, \quad \forall k \in L_{i}+1 \cdots T-\mathrm{DT}_{i}+1, \\
\sum_{n=k}^{T}\left\{1-v_{i}(n)-\left[v_{i}(k-1)-v_{i}(k)\right]\right\} \geq 0, \quad \forall i \in I, \quad \forall k \in K-\mathrm{DT}_{i}+2 \cdots K,
\end{gathered}
$$

where $L_{i}$ is the number of initial periods during which unit $i$ must be offline. $L_{i}$ is mathematically expressed as $L_{i}=\min \left\{K,\left[\mathrm{DT}_{i}-S_{i}(0)\right] \cdot\left[1-V_{i}(0)\right]\right\}$.

\subsubsection{Spinning Reserve}

$$
0 \leq \sum_{i=1}^{I} \mathrm{SR}_{i}(k) \leq \mathrm{SR}_{\operatorname{Max}}, \quad \forall k \in K .
$$

The complete procedure of spinning reserve calculation is stated in Section 3. 


\subsection{Security Constraints}

\subsubsection{System Real Power Balance}

$$
\sum_{i \in I} P_{i}(k)+P^{\mathrm{WH}}(k)+P^{\mathrm{WF}}(k)=D(k)+\mathrm{SR}(k), \quad \forall k \in K .
$$

\subsubsection{Power Fow Limit}

$$
-\mathrm{DF}_{E}^{\mathrm{Max}}(k) \leq \mathrm{DF}_{E}(k) \leq \mathrm{DF}_{E}^{\mathrm{Max}}(k), \quad \forall k \in K
$$

\subsection{Probabilistic Model of a Wind Farm Output Power}

The output power of a wind turbine generator is nonlinearly related to the wind speed. A schematic of wind turbine output is depicted in Figure 4. Output power of a wind turbine unit can be approximated by the following expression [20]:

$$
P^{\text {Wind }}= \begin{cases}0 & V \leq V_{\mathrm{ci}} \\ \left(A+B V+C V^{2}\right) & V_{\mathrm{ci}}<V \leq V_{r} \\ P_{r} & V_{r} \leq V<V_{\mathrm{co}} \\ 0 & V \geq V_{\mathrm{co}}\end{cases}
$$

where $A, B$, and $C$ are related to $V_{\mathrm{ci}}$ and $V_{r}$ as it is stated in the following equations:

$$
\begin{gathered}
A=\frac{1}{\left(V_{\mathrm{ci}}-V_{r}\right)^{2}}\left[V_{\mathrm{ci}}\left(V_{\mathrm{ci}}+V_{r}\right)-4\left(V_{\mathrm{ci}} \times V_{r}\right)\left[\frac{V_{\mathrm{ci}}+V_{r}}{2 V_{\mathrm{ci}}}\right]^{3}\right], \\
B=\frac{1}{\left(V_{\mathrm{ci}}-V_{r}\right)^{2}}\left[4\left(V_{\mathrm{ci}}+V_{r}\right)\left[\frac{V_{\mathrm{ci}}+V_{r}}{2 V_{r}}\right]^{3}-\left(3 V_{\mathrm{ci}}+V_{r}\right)\right], \\
C=\frac{1}{\left(V_{\mathrm{ci}}-V_{r}\right)^{2}}\left[2-4\left[\frac{V_{\mathrm{ci}}+V_{r}}{2 V_{r}}\right]^{3}\right] .
\end{gathered}
$$

In this paper, the velocity of wind in eight different directions and their associated times of occurrence for six years in Siahpoosh are used. This wind farm has 50 wind turbines that capacity and force outage rate of each generator are $0.1 \mathrm{MW}$ and 0.02 , respectively. The output of a wind farm that has $N_{W T}$ turbines is calculated as follows:

$$
P^{\mathrm{WF}}(k)=P^{\mathrm{Wind}}(k) A^{\mathrm{Wind}} \eta N_{\mathrm{WT}} \text {. }
$$




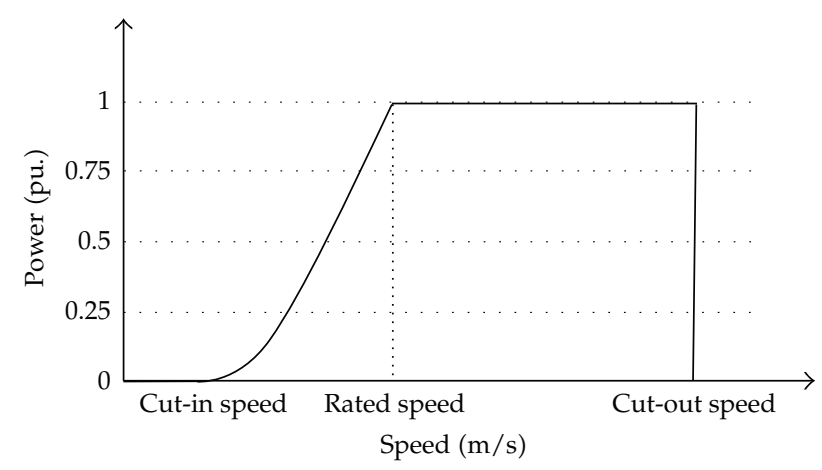

Figure 4: A schematic of wind turbine output.

The probability of different output of wind farm is stated in Table 1.

The expected average power of wind farm during a period of 24 hours is given in Table 2 .

\section{Solution Method}

In this section, the procedure of minimizing total cost of DISCO considering security and reliability indices is proposed.

\subsection{Security-Constrained Unit Commitment Based on Benders Decomposition Method}

The first part of the solving procedure is unit commitment problem with DC load flow constraints. Unit commitment in electric power systems is to optimize generating resources to supply system load while satisfying prevailing constraints, such as minimum on/off time, ramping up/down, minimum/maximum generating capacity, and fuel limit [21].

The network model is also included to consider load flow limits which is based on a DC load flow. In general, the UC problem falls into the category of large-scale and nonconvex problems that are extremely difficult to solve in an accurate and efficient way. In light of the need of more efficient tools to support decisions for resource scheduling in the new competitive business environment, the Benders decomposition method has attracted more attention in recent years $[15,22,23]$.

This approach decomposes UC into a master problem and a subproblem. The master problem is an integer programming, and subproblem is a linear programming. The master problem applies integer programming method to solve UC and find proper on/off states of the units, and the subproblem uses this solution to form appropriate cuts and adds them to the master problem for solving the next iteration of UC. All continuous variables are considered in subproblem. The iterative process will continue until a predefined gap is obtained and a converged optimal solution is found. The procedure of Benders decomposition is stated in the appendix. In the proposed decomposition approach, both subproblem and master problem would have constraints. Since the objective function and constraints of the master problem are modified in a way that there exists no continuous 
Table 1: Wind farm probability distribution for each hour.

\begin{tabular}{|c|c|c|c|c|c|c|c|c|c|c|}
\hline \multirow{2}{*}{ Hour } & \multicolumn{10}{|c|}{ Probability of occurrence } \\
\hline & $5.00 \mathrm{MW}$ & 4. $40 \mathrm{MW}$ & $85 \mathrm{MW}$ & $30 \mathrm{MW}$ & 2 & $2.20 \mathrm{~N}$ & $1.65 \mathrm{MW}$ & & & 0.00 \\
\hline 1 & 0.818548 & 0.020327 & 0.015153 & 0.023166 & 0.018659 & 0.028228 & 0.016889 & 0.020518 & 0.022072 & 0.016439 \\
\hline 2 & 7695 & 0.015938 & 0.019718 & 0.032179 & 0.025176 & & & & 0.021826 & 0304 \\
\hline 3 & 746 & 0.022605 & 0.026773 & 0.02827 & 0.018412 & & 0.024797 & 0.02426 & 0.014111 & 0.024897 \\
\hline 4 & 385 & 0.01 & 0.029188 & 0.035746 & 0.016951 & 0.034347 & 0.025023 & 0.02228 & 0.027531 & 0.025067 \\
\hline 5 & 0.746459 & 0.024967 & 0.037105 & 0.023652 & 0.020331 & 0.029074 & 0.029396 & 0.029236 & 0.029355 & 0.030423 \\
\hline 6 & 0.746459 & 0.024967 & 0.037105 & 0.023652 & 0.020331 & 0.029074 & 0.029396 & 0.029236 & 0.029355 & 4423 \\
\hline 7 & 0.725218 & 0.028932 & 0.035601 & 0.031239 & 0.028772 & 0.0234 & 0.029965 & 0.025509 & 0.036348 & 0.035015 \\
\hline 8 & 0.706415 & 0.035451 & 0.027232 & 0.033117 & 0.036965 & 0.033558 & 0.025264 & 0.039471 & 0.032951 & 9577 \\
\hline 9 & 0.688664 & 0.025491 & 0.039018 & 0.036956 & 0.049526 & & 0.028861 & & 686 & 679 \\
\hline 10 & 0.687112 & 0.031644 & 0.038404 & 0.043661 & 0.037484 & 0.040602 & 0.02828 & 0.025189 & 0.038176 & \\
\hline 11 & 0.69 & 0.04 & 0.04 & 0.027913 & 0.033965 & 0.02 & 0.02 & & 0.0 & 259 \\
\hline 12 & 0.66 & 0.03 & 0.03 & 0.039759 & 0.039677 & 0.03 & 0.036 & 0.03 & 0.04 & 0.02 \\
\hline 13 & 0.680014 & 0.02 & 0.021978 & 0.038813 & 0.040829 & 0.033277 & 0.033726 & 578 & 853 & 0.04749 \\
\hline 14 & 0.694954 & 0.026239 & 0.033476 & 0.033375 & 0.026873 & 0.042714 & 0.033401 & 0.042306 & 0.033018 & 0.033644 \\
\hline 15 & 0.681595 & 0.027552 & 0.026122 & 0.031481 & 0.034156 & 0.03965 & 0.046043 & 0.027637 & 0.031701 & 0.054061 \\
\hline 16 & 0.669687 & 0.041023 & 0.030866 & 0.033923 & 0.036513 & 0.031527 & 0.046212 & 0.043174 & 0.032458 & 0.034618 \\
\hline 17 & 0.709901 & 0.029333 & 0.034226 & 0.032659 & 0.038942 & 0.027333 & 0.029574 & 0.027794 & 0.039274 & 0.030963 \\
\hline 18 & 0.7336701 & 0.028382 & 0.024214 & 0.033826 & 0.026897 & 0.028144 & 0.032793 & 0.030391 & 0.036497 & 0.025187 \\
\hline 19 & 0.7378926 & 0.031653 & 0.020864 & 0.027788 & 0.0335 & 0.027692 & 0.038773 & 0.023567 & 0.033453 & 0.024733 \\
\hline 20 & 0.7436435 & 0.027286 & 0.024931 & 0.025754 & 0.025641 & 0.030585 & 0.027332 & 0.031297 & 0.026752 & 0.036778 \\
\hline 21 & 0.756614 & 0.033106 & 0.020549 & 0.034601 & 0.026051 & 0.019852 & 0.018945 & 0.031869 & 0.03031 & 0.028103 \\
\hline 22 & 0.7854144 & 0.026957 & 0.027744 & 0.023654 & 0.024437 & 0.02271 & 0.021103 & 0.021168 & 0.019273 & 0.027539 \\
\hline 23 & 0.793456 & 0.023663 & 0.025487 & 0.022799 & 0.025087 & 0.027165 & 0.021596 & 0.017834 & 0.022994 & 0.019918 \\
\hline 24 & 0.8180212 & 0.018876 & 0.021151 & 0.016845 & 0.023261 & 0.021499 & 0.021484 & 0.018685 & 0.018704 & 0.021473 \\
\hline
\end{tabular}

Table 2: Expected average power output of a wind farm.

\begin{tabular}{lccc}
\hline Hour & Capacity in (MW) & Hour & Capacity in (MW) \\
\hline 1 & 4.492956 & 13 & 4.044532 \\
2 & 4.465466 & 14 & 4.116921 \\
3 & 4.416840 & 15 & 4.038627 \\
4 & 4.341394 & 16 & 4.071079 \\
5 & 4.279737 & 17 & 4.186309 \\
6 & 4.279737 & 18 & 4.241576 \\
7 & 4.221640 & 19 & 4.262340 \\
8 & 4.180897 & 20 & 4.251286 \\
9 & 4.139469 & 21 & 4.320334 \\
10 & 4.154502 & 22 & 4.416424 \\
11 & 4.148085 & 23 & 4.441409 \\
12 & 4.087347 & 24 & 4.487735 \\
\hline
\end{tabular}

variable in this problem, the solution will be found faster than conventional mixed integer linear programming (MILP). 
The flowchart of the Benders decomposition procedure followed in this paper includes the steps illustrated in Figure 5.

\subsubsection{Subproblem}

The objective function of subproblem is as (3.1) in which the integer part is fixed

$$
\mathrm{SP}=\min \sum_{k=1}^{K} \sum_{i=1}^{I}\left[A_{i}+\sum_{l=1}^{\mathrm{NL}} F_{l, i} \delta_{l, i}+\mathrm{CD}_{i}(k)+\mathrm{CS}_{i}(k)\right]+\sum_{k=1}^{K} C\left(P^{\mathrm{WH}}(k)\right) .
$$

The subproblem will be solved subject to linear model of power generation (2.6), ramp-up and ramp-down constraints (2.7)-(2.9), power balance (2.18), and DC power flow equations (2.19).

\subsubsection{Relaxed Subproblem}

If the subproblem is not feasible, the subproblem will be relaxed in order to make the subproblem feasible. The formulation of the relaxed subproblem is stated as follows:

$$
\begin{gathered}
\min \sum_{i=1}^{I} \sum_{\mathrm{rs}=1}^{5} R_{i, r s}, \\
p_{i}(k)-R_{i, 1} \geq \underline{P}_{i}(k) \widehat{v}_{i}(k), \\
\bar{p}_{i}(k)-R_{i, 2} \leq \bar{P}_{i}(k) \widehat{v}_{i}(k), \\
\bar{p}_{i}(k)-p_{i}(k-1)-R_{i, 3} \leq+\bar{P}_{i}\left[1-\widehat{v}_{i}(k)\right]+\mathrm{SU}_{i}\left[\widehat{v}_{i}(k)-\widehat{v}_{i}(k-1)\right]+\mathrm{RU}_{i} \widehat{v}_{i}(k-1), \\
\bar{p}_{i}(k)-R_{i, 4} \leq \bar{P}_{i} \widehat{v}_{i}(k+1)+\mathrm{SD}_{i}\left[\widehat{v}_{i}(k)-\widehat{v}_{i}(k+1)\right]+\mathrm{RU}_{i} \widehat{v}_{i}(k-1), \\
p_{i}(k-1)-p_{i}(k)-R_{i, 5} \leq \mathrm{SD}_{i}\left[\widehat{v}_{i}(k-1)-\widehat{v}_{i}(k)\right]+\bar{P}_{i}\left[1-\widehat{v}_{i}(k-1)\right]+\mathrm{RD}_{i} \widehat{v}_{i}(k) .
\end{gathered}
$$

After solving the relaxed subproblem and finding the dual variables, $\lambda_{i, \mathrm{rs}}^{\mathrm{rx}}$, a cut (3.3) is added to the master problem,

$$
\begin{aligned}
\operatorname{Cut}^{\mathrm{rx}}= & \lambda_{i, 1}^{\mathrm{rx}}(k)\left[\underline{P}_{i}(k) v_{i}(k)-p_{i}(k)\right]+\lambda_{i, 2}^{\mathrm{rx}}(k)\left[\bar{p}_{i}(k)-\bar{P}_{i}(k) v_{i}(k)\right] \\
& +\lambda_{i, 3}^{\mathrm{rx}}(k)\left[\bar{p}_{i}(k)-p_{i}(k-1)-\bar{P}_{i}\left[1-v_{i}(k)\right]-\mathrm{SU}_{i}\left[v_{i}(k)-v_{i}(k-1)\right]-\mathrm{RU}_{i} v_{i}(k-1)\right] \\
& +\lambda_{i, 4}^{\mathrm{rx}}(k)\left[\bar{p}_{i}(k)-\bar{P}_{i} v_{i}(k+1)-\mathrm{RU}_{i} v_{i}(k-1)-\mathrm{SD}_{i}\left[v_{i}(k)-v_{i}(k+1)\right]\right] \\
& +\lambda_{i, 5}^{\mathrm{rx}}(k)\left[p_{i}(k-1)-p_{i}(k)-\bar{P}_{i}\left[1-v_{i}(k-1)\right]-\mathrm{SD}_{i}\left[v_{i}(k-1)-v_{i}(k)\right]\right] \leq 0 .
\end{aligned}
$$




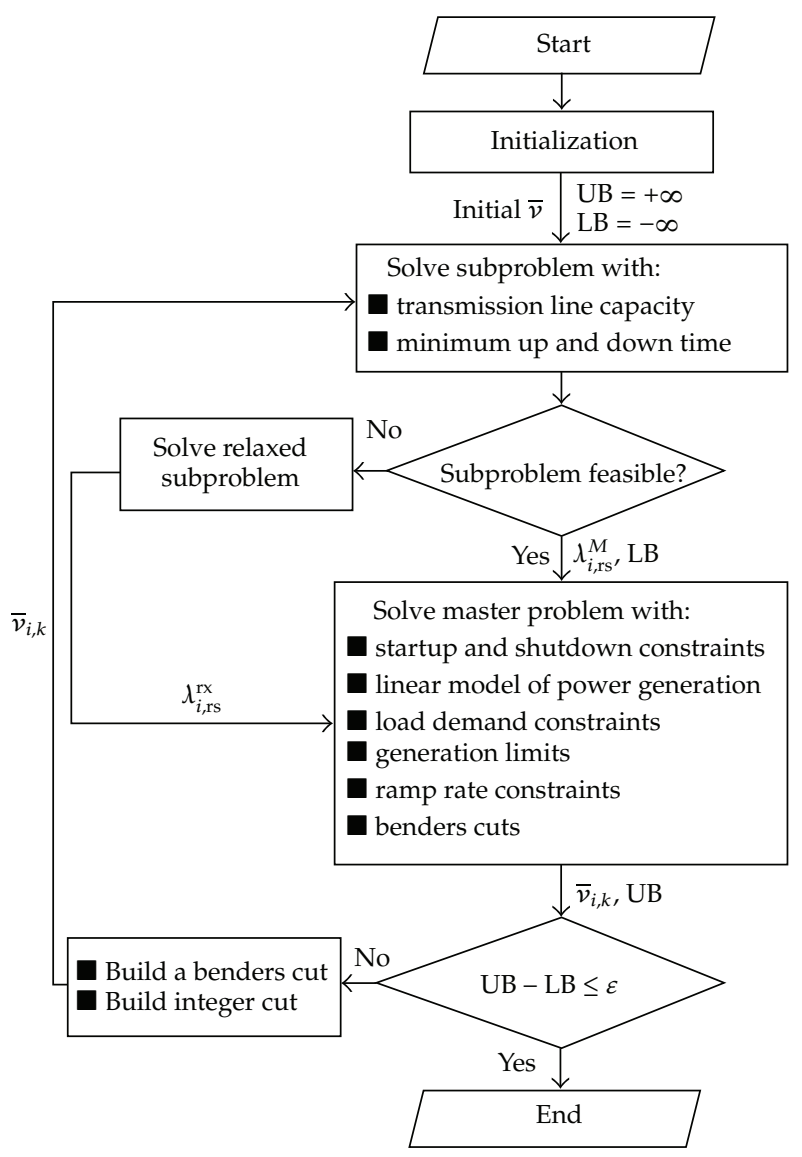

Figure 5: General procedure of Benders decomposition implementation.

\subsubsection{Master Problem}

The objective function of the master problem is as (3.1) in which the continuous variables are fixed. Constraints regarding the master problem are startup and shutdown constraints (2.10) and (2.11) and minimum up time (2.12)-(2.14) and minimum downtime constraints (2.15)-(2.17).

A Benders cut (3.4) is added to the master problem while the master problem does not converge.

The master problem will be solved by either (3.3) or (3.4), and the optimization problem will be terminated while $\mathrm{UB}-\mathrm{LB} \leq \varepsilon$.

\subsection{Calculation of Reliability Indices}

Reliability constraints are implemented in order to achieve a reliable unit commitment schedule. There are two ways to consider reliability constraints. The first method is to 
consider reliability indices implicitly as unit commitment's constraints in a mixed integer linear format and calculate unit commitment and reliability indices simultaneously,

$$
\begin{aligned}
\mathrm{Cut}^{M}= & \lambda_{i, 1}^{M}(k)\left[\underline{P}_{i}(k) v_{i}(k)-p_{i}(k)\right]+\lambda_{i, 2}^{M}(k)\left[\bar{p}_{i}(k)-\bar{P}_{i}(k) v_{i}(k)\right] \\
& +\lambda_{i, 3}^{M}(k)\left[\bar{p}_{i}(k)-p_{i}(k-1)-\bar{P}_{i}\left[1-v_{i}(k)\right]-\mathrm{SU}_{i}\left[v_{i}(k)-v_{i}(k-1)\right]-\mathrm{RU}_{i} v_{i}(k-1)\right] \\
& +\lambda_{i, 4}^{M}(k)\left[\bar{p}_{i}(k)-\bar{P}_{i} v_{i}(k+1)-\mathrm{RU}_{i} v_{i}(k-1)-\mathrm{SD}_{i}\left[v_{i}(k)-v_{i}(k+1)\right]\right] \\
& +\lambda_{i, 5}^{M}(k)\left[p_{i}(k-1)-p_{i}(k)-\bar{P}_{i}\left[1-v_{i}(k-1)\right]-\mathrm{SD}_{i}\left[v_{i}(k-1)-v_{i}(k)\right]\right]
\end{aligned}
$$

This method has been implemented in [11] for one hour. The result of this method is optimum; however, it is impractical for a 24-hour period because it is computationally extensive. Another method which is used in this paper is to calculate the reliability indices sequentially. Since computating reliability indices in DELPHI-executable format are very fast, the authors programmed reliability indices in DELPHI language.

\subsection{LOLP Calculation}

Once the status of each unit is determined by unit commitment, the LOLP is checked for each hour:

$$
\begin{gathered}
\operatorname{LOLP}(k)=\sum_{s=1}^{S} \operatorname{Pr}_{s} \operatorname{LOSS}_{i}(k), \quad k \in K, \\
\operatorname{LOLP}(k) \leq \operatorname{LOLP}_{\text {Max }},
\end{gathered}
$$

where

$$
\operatorname{LOSS}_{i}(k)= \begin{cases}1, & C_{R i} \leq \operatorname{Load}(k) \\ 0, & \text { Otherwise }\end{cases}
$$

If (3.6) is not satisfied in hour $k$, the unit commitment result will be rejected. In another word, the committed units do not guarantee a reliable system. So an integer cut is added to unit commitment constraints. Then unit commitment should be calculated again. Besides, the amount of spinning reserve will be determined as follows:

$$
\begin{gathered}
\operatorname{SR}(k)=\left(\sum_{i=1}^{I}\left(P_{i}^{\operatorname{Max}}(k) v_{i}(k)\right)-\sum_{i=1}^{I}\left(P_{i}(k) v_{i}(k)\right)\right)+\varepsilon, \\
0 \leq \operatorname{SR}(k) \leq \operatorname{SR}^{\operatorname{Max}}(k) .
\end{gathered}
$$

This iterative process continues until (3.8) is satified for a 24-hour period. 


\subsection{Expected Interruption Cost}

Most methods that have been proposed for calculating expected interruption cost were based on a combination of a cost model and a set of reliability indices. An important factor in calculating expected interruption cost is that cost of a service interruption is much higher for some customers than others. Therefore, some customers are willing to pay more for higher reliable level, while others would accept less reliable system for reduction in their electricity cost. So in this paper, the value of the loss load (VOLL) is considered the same for all customers. The expected interruption cost is as follows:

$$
C_{\mathrm{EIC}}=\mathrm{EENS} * \mathrm{VOLL},
$$

where EENS is the energy is expected energy that is not going to be supplied. Different methods have been implemented to calculate VOLL. In this work, VOLL is $\$ 1000 / \mathrm{MWh}$.

Since expected interruption cost is related to the configuration of committed units, different units' combination leads to different expected interruption cost. In this paper, the first 5 total costs are saved.

\section{Case Study}

The proposed algorithm has been implemented in GAMS and DELPHI. The effectiveness of the proposed method was initially tested on an eight-bus test system in which the reliability indices were not included. The result of the proposed method has been compared with [24]. In the second test system, we modified the 33-bus distribution test system [25] by adding three DG units owned by disco and a wind farm. Two different scenarios are implemented to examine the operational issues of proposed method.

\subsection{Case Study 1}

In this section, the convergence speed of proposed method is compared with an eight-bus test system. The single line diagram of the test system is given in Figure 6.

In this first case, the reliability indices are not considered and only the effectiveness of benders decomposition in solving unit commitment, in which binary variables are only on/off of units, is examined. In this case, the deterministic spinning reserve is considered. The deterministic spinning reserve is set to 10 percent of total load. Table 3 shows the simulation result of the first case study. The total cost of proposed method $\$ 80982.65$ is the same as [24]; however, the execution time is improved by \%33.2. The improvement is due to the decomposition of the unit commitment problem into two stages as well as reformulating the unit commitment in a way that only binary variables associated with on/off of units are considered.

The proposed method is applied to a modified 33-distribution test system. The single line test system and its data are in Figure 7. 


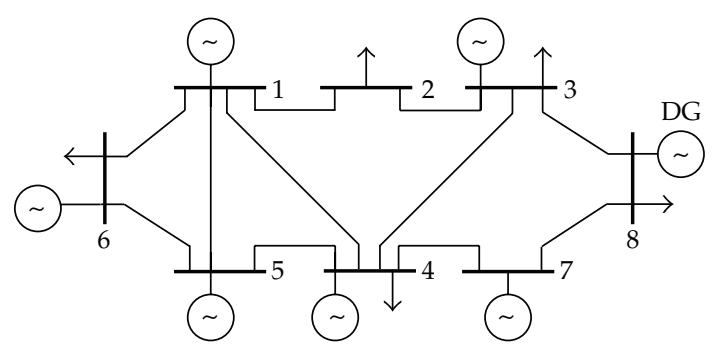

Figure 6: An eight bust test system.

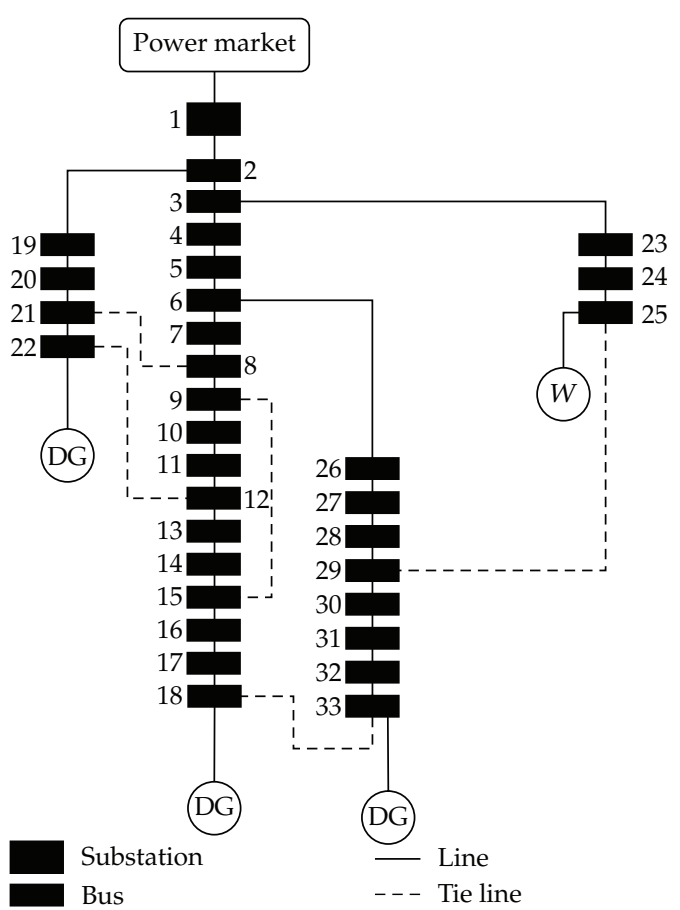

Figure 7: The modified 33-bus distribution test system.

Table 3: Simulation result of the eight bus test system.

\begin{tabular}{lcc}
\hline \multicolumn{1}{c}{ Total cost $=\$ 80981.65$} \\
\hline Unit \\
\hline G1 \\
G2 \\
G3 \\
G4
\end{tabular}

\subsection{Case Study 2}

Three DG units and a wind farm owned by the disco are supposed to be connected at the end of distribution buses. It should be noted that, the locations of DGs in this work are absolutely 
Table 4: Hourly expected average power output of wind farm and market price.

\begin{tabular}{lccccc}
\hline Hour & Load $(\mathrm{MW})$ & Market price $(\$ / \mathrm{MW})$ & Hour & Load $(\mathrm{MW})$ & Market price $(\$ / \mathrm{MW})$ \\
\hline 1 & 10.30 & 40.2372 & 13 & 11.50 & 62.1350 \\
2 & 10.50 & 43.8869 & 14 & 11.50 & 62.1350 \\
3 & 10.43 & 42.6095 & 15 & 11.20 & 56.6606 \\
4 & 10.44 & 42.7920 & 16 & 11.20 & 56.6606 \\
5 & 10.43 & 42.6095 & 17 & 11.20 & 56.6606 \\
6 & 10.60 & 45.7117 & 18 & 11.20 & 56.6606 \\
7 & 10.70 & 47.5365 & 19 & 10.95 & 52.0985 \\
8 & 10.80 & 49.3613 & 20 & 10.80 & 49.3613 \\
9 & 11.10 & 54.8358 & 21 & 10.70 & 47.5365 \\
10 & 11.10 & 54.8358 & 22 & 10.40 & 42.0620 \\
11 & 11.50 & 62.1350 & 23 & 10.30 & 40.2372 \\
12 & 11.60 & 63.9599 & 24 & 10.30 & 40.2372 \\
\hline
\end{tabular}

selected arbitrary. The two of them, at the buses-18 and 33, are $5 \mathrm{MW}$ capacities each, while the last one at bus-22 is $4 \mathrm{MW}$ capacity. The output of wind farm, which is connected to bus25 , is illustrated in Table 2. The hourly demands as well as their associated price are stated in Table 4. The tie lines are not put into practice in this paper. The disco is able to purchase energy from wholesalers at power market which is connected at bus-1.

The total cost of this case is $\$ 6616.282$. It is clear that when the cost of electricity is low in some hours, the disco tends to purchase energy from market instead of using DG-2 or DG-3. Three DG units and a wind farm owned by the disco are supposed to be connected at the end of distribution buses. It should be noted that the locations of DGs in this work are absolutely selected arbitrary. The two of them, at the buses-18 and 33, are $5 \mathrm{MW}$ capacities each, while the last one at bus-22 is $4 \mathrm{MW}$ capacity. The output of wind farm, which is connected to bus-25, is illustrated in Table 2 . The hourly demands as well as their associated price are stated in Table 4 . The tie lines are not put into practice in this paper. The disco is able to purchase energy from wholesalers at power market which is connected at bus- 1 .

In order to discuss the efficiency of proposed method in details, we consider the following three cases.

Case 1. Base case.

Case 2. Base case $+\mathrm{DC}$ power flow + deterministic spinning reserve.

Case 3. Base case + DC power flow + reliability constraints + probabilistic spinning reserve.

\subsubsection{Case 1}

In this case, only a two-level unit commitment is considered. The disco provides its needed energy from wind farm, its own DG units, and power market. All generators are supposed to be ON for the first hour. The DG-1 is the cheapest DG unit while DG-2 is the most expensive one. The simulation result of this case is depicted in Table 5 . 
Table 5: Simulation result of Case 1.

\begin{tabular}{|c|c|c|c|c|c|}
\hline \multicolumn{6}{|c|}{ Total cost $=\$ 6,616.282$} \\
\hline Time & DG-1 & DG-2 & DG-3 & WF & PM \\
\hline 1 & 3.807 & 1.000 & 1.000 & 4.492 & 0.000 \\
\hline 2 & 5.000 & 0.000 & 0.000 & 4.465 & 1.035 \\
\hline 3 & 5.000 & 0.000 & 0.000 & 4.416 & 1.013 \\
\hline 4 & 5.000 & 0.000 & 0.000 & 4.341 & 1.099 \\
\hline 5 & 5.000 & 0.000 & 0.000 & 4.279 & 1.150 \\
\hline 6 & 5.000 & 0.000 & 0.000 & 4.279 & 1.320 \\
\hline 7 & 5.000 & 0.000 & 0.000 & 4.221 & 1.478 \\
\hline 8 & 5.000 & 0.000 & 0.000 & 4.180 & 1.619 \\
\hline 9 & 5.000 & 0.000 & 1.250 & 4.139 & 0.710 \\
\hline 10 & 5.000 & 0.000 & 1.945 & 4.154 & 0.000 \\
\hline 11 & 5.000 & 0.000 & 2.352 & 4.148 & 0.000 \\
\hline 12 & 5.000 & 0.000 & 2.513 & 4.087 & 0.000 \\
\hline 13 & 5.000 & 0.000 & 2.455 & 4.044 & 0.000 \\
\hline 14 & 5.000 & 0.000 & 2.383 & 4.116 & 0.000 \\
\hline 15 & 5.000 & 0.000 & 2.161 & 4.038 & 0.000 \\
\hline 16 & 5.000 & 0.000 & 2.129 & 4.071 & 0.000 \\
\hline 17 & 5.000 & 0.000 & 2.014 & 4.186 & 0.000 \\
\hline 18 & 5.000 & 0.000 & 1.958 & 4.241 & 0.000 \\
\hline 19 & 5.000 & 0.000 & 1.250 & 4.262 & 0.438 \\
\hline 20 & 5.000 & 0.000 & 0.000 & 4.251 & 1.549 \\
\hline 21 & 5.000 & 0.000 & 0.000 & 4.320 & 1.380 \\
\hline 22 & 5.000 & 0.000 & 0.000 & 4.416 & 0.984 \\
\hline 23 & 5.000 & 0.000 & 0.000 & 4.441 & 0.859 \\
\hline 24 & 5.000 & 0.000 & 0.000 & 4.487 & 0.812 \\
\hline
\end{tabular}

\subsubsection{Case 2}

In this case, the unit commitment in which DC load flow equations as well as spinning reserve are included is considered. The amount of deterministic spinning reserve is set to $3 \mathrm{MW}$ for all hours. This method of calculating spinning reserve does not take into consideration the probability of occurrence of this contingency, if its probability is low, on the average, overscheduling of reserve may result, while if the probability of occurrence is high, the reserve may be insufficient [26]. The transmission constraints are also added to unit commitment constraints. The DC load flow variables are belonged to the subproblem. The total cost of this case is $\$ 12049.852$. The simulation result is stated in Table 6. It is clear that DG-2 is the most expensive units. The cost of second case is greater by $82 \%$ comparing with Case 1. This increase in cost is because of using spinning reserve as well as considering security constraints.

\subsubsection{Case 3}

In this case, we consider the complete model of DISCO in which security as well as reliability constraints are included. 
Table 6: Simulation results of Case 2.

\begin{tabular}{|c|c|c|c|c|c|}
\hline \multicolumn{6}{|c|}{ Total cost $=\$ 12,049.852$} \\
\hline Time & DG-1 & DG-2 & DG-3 & W & PM \\
\hline 1 & 5.000 & 1.000 & 3.750 & 4.492 & 1.057 \\
\hline 2 & 5.000 & 0.000 & 5.000 & 4.465 & 1.034 \\
\hline 3 & 5.000 & 0.000 & 5.000 & 4.416 & 1.013 \\
\hline 4 & 5.000 & 0.000 & 5.000 & 4.341 & 1.099 \\
\hline 5 & 5.000 & 0.000 & 5.000 & 4.279 & 1.150 \\
\hline 6 & 5.000 & 0.000 & 5.000 & 4.279 & 1.320 \\
\hline 7 & 5.000 & 0.000 & 5.000 & 4.221 & 1.478 \\
\hline 8 & 5.000 & 0.000 & 5.000 & 4.180 & 1.619 \\
\hline 9 & 5.000 & 0.000 & 5.000 & 4.139 & 1.960 \\
\hline 10 & 5.000 & 0.000 & 5.000 & 4.154 & 1.945 \\
\hline 11 & 5.000 & 0.000 & 5.000 & 4.148 & 2.352 \\
\hline 12 & 5.000 & 0.000 & 5.000 & 4.087 & 2.513 \\
\hline 13 & 5.000 & 0.000 & 5.000 & 4.044 & 2.456 \\
\hline 14 & 5.000 & 0.000 & 5.000 & 4.116 & 2.383 \\
\hline 15 & 5.000 & 0.000 & 5.000 & 4.038 & 2.161 \\
\hline 16 & 5.000 & 0.000 & 5.000 & 4.071 & 2.129 \\
\hline 17 & 5.000 & 0.000 & 5.000 & 4.186 & 2.014 \\
\hline 18 & 5.000 & 0.000 & 5.000 & 4.241 & 1.958 \\
\hline 19 & 5.000 & 0.000 & 5.000 & 4.262 & 1.688 \\
\hline 20 & 5.000 & 0.000 & 5.000 & 4.251 & 1.549 \\
\hline 21 & 5.000 & 0.000 & 5.000 & 4.320 & 1.380 \\
\hline 22 & 5.000 & 0.000 & 5.000 & 4.416 & 0.984 \\
\hline 23 & 5.000 & 0.000 & 5.000 & 4.441 & 0.859 \\
\hline 24 & 5.000 & 0.000 & 3.750 & 4.487 & 2.062 \\
\hline
\end{tabular}

In Table 7, the result of unit commitment with DC load flow considerations, spinning reserve, hourly LOLP, and total EEN are presented. As it is clear in Table 6, in some hours, DG2 which is the most expensive unit are going to be $\mathrm{ON}$ in order to improve the reliability of the system. The hourly spinning reserve which was obtained from (3.8) and (3.9) is set in a way that LOLP in all hours will be less than 0.05 . The EENS of the system is $0.742 \mathrm{MWh}$. The total cost of the system (2.2) is $\$ 9388.999$ which was improved by $0.22 \%$. Table 7 includes the first 5 EENS values and their associated total costs. As it is extracted from Table 8, the first result has the minimum total cost.

\section{Conclusion}

This paper presented a reliability-based unit commitment of a DISCO in restructured power market. The probabilistic output of the real wind farm is also used in this paper. A step-by-step procedure was stated to clarify the applicability of proposed methodology. In unit commitment formulation, only one set of binary variables, on/off of the units, was used. The Benders decomposition was used to solve the unit commitment problem. The LOLP and EENS indices were programmed in Delphi environment and linked with GAMS. The proposed algorithm was applied to two-bus test system. The numerical 
Table 7: Simulation result of Case 3.

\begin{tabular}{|c|c|c|c|c|c|c|c|}
\hline \multicolumn{4}{|c|}{ Total cost $=\$ 9388.999$} & \multicolumn{4}{|c|}{ EENS $=0.742$} \\
\hline Time & DG-1 & DG-2 & DG-3 & WF & PM & LOLP & SR \\
\hline 1 & 4.750 & 1.000 & 1.750 & 4.492 & 0.000 & 0.019 & 0.661 \\
\hline 2 & 5.000 & 0.000 & 2.004 & 4.465 & 0.000 & 0.003 & 0.968 \\
\hline 3 & 5.000 & 0.000 & 5.000 & 4.416 & 0.000 & 0.011 & 1.360 \\
\hline 4 & 5.000 & 0.000 & 2.375 & 4.341 & 0.000 & 0.014 & 1.837 \\
\hline 5 & 5.000 & 0.000 & 2.937 & 4.279 & 0.000 & 0.016 & 2.383 \\
\hline 6 & 5.000 & 0.000 & 3.534 & 4.279 & 0.000 & 0.040 & 2.969 \\
\hline 7 & 5.000 & 0.000 & 4.291 & 4.221 & 0.035 & 0.044 & 3.555 \\
\hline 8 & 5.000 & 0.000 & 5.000 & 4.180 & 0.710 & 0.023 & 4.090 \\
\hline 9 & 5.000 & 0.000 & 5.000 & 4.139 & 1.482 & 0.042 & 4.520 \\
\hline 10 & 5.000 & 0.000 & 5.000 & 4.154 & 1.746 & 0.044 & 4.800 \\
\hline 11 & 5.000 & 1.000 & 5.000 & 4.148 & 1.250 & 0.023 & 4.897 \\
\hline 12 & 5.000 & 2.000 & 5.000 & 4.087 & 0.313 & 0.013 & 4.800 \\
\hline 13 & 5.000 & 1.977 & 5.000 & 4.044 & 0.000 & 0.040 & 4.520 \\
\hline 14 & 5.000 & 1.000 & 5.000 & 4.116 & 0.474 & 0.012 & 4.089 \\
\hline 15 & 5.000 & 0.000 & 5.000 & 4.038 & 0.718 & 0.050 & 3.555 \\
\hline 16 & 5.000 & 0.000 & 5.000 & 4.071 & 0.100 & 0.047 & 2.970 \\
\hline 17 & 5.000 & 0.000 & 4.398 & 4.186 & 0.000 & 0.014 & 2.383 \\
\hline 18 & 5.000 & 0.000 & 3.797 & 4.241 & 0.000 & 0.034 & 1.837 \\
\hline 19 & 5.000 & 0.000 & 3.049 & 4.262 & 0.000 & 0.010 & 1.361 \\
\hline 20 & 5.000 & 0.000 & 2.500 & 4.251 & 0.018 & 0.040 & 0.969 \\
\hline 21 & 5.000 & 0.000 & 1.250 & 4.320 & 0.793 & 0.050 & 0.662 \\
\hline 22 & 5.000 & 0.000 & 0.000 & 4.416 & 1.419 & 0.049 & 0.435 \\
\hline 23 & 5.000 & 0.000 & 0.000 & 4.441 & 1.133 & 0.034 & 0.274 \\
\hline 24 & 5.000 & 0.000 & 0.000 & 4.487 & 0.979 & 0.013 & 0.166 \\
\hline
\end{tabular}

Table 8: The first 5 results.

\begin{tabular}{lccc}
\hline No. & EENS & OC & TC \\
\hline 1 & 0.742 & 8646.999 & 9388.999 \\
2 & 0.811 & 9023.263 & 9834.263 \\
3 & 0.834 & 8987.215 & 9821.215 \\
4 & 0.891 & 9124.752 & 10015.752 \\
5 & 0.954 & 9937.542 & 10891.542 \\
\hline
\end{tabular}

results demonstrated the efficiency of proposed method. The proposed model is more comprehensive in comparison with other related done works.

\section{Appendix}

\section{Unit Commitment Based on Benders Decomposition}

The unit commitment problem is a MILP in which the master problem includes on/off of generators and some constraints that include only binary variables while the subproblem 
includes continuous variables of generation of each unit and other constraints that include continuous variables. A MILP is assumed as follows:

$$
\begin{gathered}
\min C y+f(x), \\
h(x)=0, \quad g(x)=0, \\
A x+B y \leq b_{1}, \quad D x+E y \leq b_{2}, \\
G y \leq a, x \in R^{n}, \quad y \in\{0,1\},
\end{gathered}
$$

where $x$ and $y$ represent linear and integer variables, respectively. Each master problem provides a lower bound which represent and optimistic estimate of the original problem solution. Each subproblem provides an upper bound with respect to $\bar{y}$. The iterative "testing" of difference between upper bound and lower bound helps master problem to learn about the problem. As Benders' cuts are added to the master problem, the objective function, which is an upper bound on the original problem optimal solution, is monotonically decreasing. The Benders' cuts provide an approximation of the subproblem objective function value for different sets of $\bar{x}$ in solution. Nevertheless, each cut provides some information about the influence of each $x$ on the problem solution. When the optimistic and conservative bound coverage, the optimal solution of original problem is represented as the subproblem solution which generated the last conservative bound, combined with the sets of $\bar{x}$ values which generated that solution. The Benders algorithm as implemented is the following.

Step 1. Initializing:

$$
\mathrm{UB}=+\infty, \quad \mathrm{LB}=-\infty, \quad \varepsilon=\mathrm{UB}-\mathrm{LB} .
$$

Step 2. Solve subproblem. The initial subproblem is solved after adjusting the subproblem to reflect the $\bar{y}$ vector's impact on constraint right hand sides,

$$
\begin{aligned}
& \text { Solve } \min C \bar{y}+f(x), \\
& h(x)=0, \\
& g(x) \leq 0, \\
& A x \leq b_{1}-B \bar{y}, \\
& D x=b_{2}-E \bar{y},
\end{aligned}
$$

where $\lambda$ are the duals for inequalities $A x \leq b_{1}-B \bar{y}$. 
Step 3. Comparing. The subproblem-objective function plus $B \bar{y}$ is compared to the previous conservative bound. If this sum represents an improvement over bound, the current $\bar{y}$ and associated subproblem solutions are saved as the incumbent solution

$$
\begin{gathered}
\mathrm{UB}=Z_{\mathrm{NLP}}, \\
x^{*}=\bar{x}, \\
y^{*}=\bar{y} .
\end{gathered}
$$

The difference between conservative and optimistic bound is then compared to convergence tolerance $(\varepsilon)$. If bound difference is less than or equal to $\varepsilon$, go to Step 7 . If not, the iteration number is incremented by 1 and new Benders' cut is formed which is then added as another constraints in the master problem:

$$
Z_{\mathrm{MIP}} \geq C y+f(\bar{x})+\lambda\left[b_{1}-B y-A \bar{x}\right]
$$

Step 4. Solve Master Problem:

$\min Z_{\mathrm{MIP}}$

s.t. cuts from before iteration

Gy $\leq a$

$$
y \in\{0,1\} \text {. }
$$

Step 5. New Optimistic Bound. The master problem-objective function value becomes new optimistic bound. Then it is checked for problem convergence. If the difference is less than or equal to tolerance, go to Step 7 . Else, based on the new master solution, $b_{1}-B y$ right hand side of the subproblem is computed.

Step 6. Based on the $\bar{y}$ from Step 4 , the subproblem is solved. Then return to Step 3.

Step 7. Dual variables. The dual variables are calculated for the optimal solution. The incumbent solution from Step 3 is then printed and the procedure is terminated.

\section{Units}

Constants

$A^{\text {Wind: }}$ Constant of wind farm

$\eta$ : $\quad$ Coefficient of a wind farm output

$N_{\text {WT }}$ : Number of wind turbines

$a_{i}, b_{i}, c_{i}$ : Coefficients of quadratic production cost function of unit $i$

$\operatorname{Pr}_{s}$ : Probability of $s$ th state

S: $\quad$ Number of states 
$H_{i}^{k}: \quad$ Startup constant of unit $i$

$A, B, C$ : Coefficients of wind turbine output

$\mathrm{NL}_{i}$ : $\quad$ Number of the segments of the piecewise linear production cost function

$\bar{P}_{i}: \quad$ Capacity of unit $i$

$F_{l i}$ : Slope of block $l$ of the piecewise linear production cost function of unit $i$

$C_{i}: \quad$ Shutdown cost of unit $i$

$T_{l i}$ : $\quad$ Upper limit of block $l$ of the piecewise linear production cost function of unit $i$

$\underline{P}_{i}: \quad$ Minimum power output of unit $i$

$P_{i}^{\mathrm{Max}}: \quad$ Maximum power of power plant

$P^{\text {wind }}(k)$ : Power output of a wind turbine in hour $k$

$P^{\mathrm{WF}}(k)$ : Power output of a wind farm in hour $k$

$P_{\text {Min }}^{\mathrm{WH}}(k)$ : Minimum power allowed from wholesaler

$P_{\mathrm{Max}}^{\mathrm{WH}}(k)$ : Maximum power allowed from wholesaler

$\mathrm{SR}_{\mathrm{MAX}}:$ Maximum spinning reserve

$P_{r}$ : $\quad$ Rated output power of a wind turbine

$\mathrm{DF}_{E}^{\mathrm{Max}}(k)$ : Maximum power flow limit of line $\mathrm{E}$ in hour $k$

$\mathrm{RU}_{i}: \quad$ Ramp-up limit of unit $i$

$\mathrm{SD}_{i}$ : $\quad$ Shutdown ramp rate of $i$ th unit

$S$ : $\quad$ Iteration number for saving EENS

$\mathrm{SR}_{\max }: \quad$ Maximum value of spinning reserve

$\mathrm{SU}_{i}: \quad$ Startup ramp rate of $i$ th unit

UB: $\quad$ Upper bound of master problem in Benders decomposition method

LB: $\quad$ Lower bound of master problem in Benders decomposition method

$V_{\mathrm{ci}}: \quad$ Cut-in speed of a wind turbine

$V_{r}: \quad$ Rated speed of a wind turbine

$V_{\text {co }}: \quad$ Cut-out speed of a wind turbine

$\widehat{v}(k): \quad$ Fixed binary variables

$U_{i}^{0}$ : $\quad$ Number of periods unit $i$ has been offline prior to the first period of time span

$V_{i}^{0}: \quad$ Initial commitment state of unit $i$

$S_{i}(0)$ : $\quad$ Number of periods unit $i$ has been offline prior to the first period of time span

UT: $\quad$ Minimum up time of unit $i$

DT: $\quad$ Minimum down time of unit $i$

LOLP $_{\text {Max }}$ : Maximum allowed LOLP.

\section{Variables}

OC: $\quad$ Operating cost of DISCO

$C_{\mathrm{TC}}: \quad$ Total cost of DISCO

$C_{\mathrm{SR}}(k)$ : Cost of spinning reserve in hour $k$

$C_{\text {EIC }}$ : Expected interruption cost

$C_{i}(k)$ : Cost of operation of unit $i$ in hour $k$

$\mathrm{CS}_{i}(k)$ : Startup cost of $i$ th unit

$\mathrm{CD}_{i}(k)$ : Shutdown cost of $i$ th unit

$\mathrm{DF}_{l}(k)$ : DC power flow of line $E$ in hour $k$

$\mathrm{SR}_{i}(k)$ : Spinning reserve in hour $k$

$p_{i}(k)$ : Power output of unit $i$ in hour $k$ 
$\bar{p}_{i}(k): \quad$ Maximum available power output of unit $i$ in hour $k$

$v_{i}(k)$ : $\quad$ Binary variables that are equal to 1 if unit $i$ is online in period $k$ and 0 otherwise

$\delta_{l, i}(k)$ : $\quad$ Power produced in block $l$ of the piecewise linear production cost function of unit $i$ in period $k$

$R_{i, \mathrm{rs}}: \quad$ Relaxed objective function in Benders decomposition

$\operatorname{LOLP}(k)$ : LOLP of hour $k$

$\lambda_{i, \mathrm{rs}}^{M}(k)$ : Dual variables of unit $i$ related to equation rs of subproblem

$\lambda_{i, \mathrm{rs}}^{\mathrm{rx}}(k)$ : Dual variables of unit $i$ related to equation rs of relaxed subproblem

SP: $\quad$ Objective function of subproblem

Cut $_{M}: \quad$ Benders cut of master problem

Cut $^{\mathrm{rx}}$ : Benders cut of relaxed subproblem.

Sets

I: Set of indexes of the generating units

$K$ : Set of indexes of the time periods

rs: Counter in Benders decomposition formulation.

\section{References}

[1] A. A. S. Algarni and K. Bhattacharya, "A generic operations framework for discos in retail electricity markets," IEEE Transactions on Power Systems, vol. 24, no. 1, pp. 356-367, 2009.

[2] "Organization for economic co-operation and development," in Distributed Generation in Liberalized Electric Markets, International Energy Agency, 2002.

[3] R. Palma-Behnke, J. L. A. Cerda, L. S. Vargas, and A. Jofré, "A distribution company energy acquisition market model with integration of distributed generation and load curtailment options," IEEE Transactions on Power Systems, vol. 20, no. 4, pp. 1718-1727, 2005.

[4] H. Li, Y. Li, and Z. Li, "A multiperiod energy acquisition model for a distribution company with distributed generation and interruptible load," IEEE Transactions on Power Systems, vol. 22, no. 2, pp. 588-596, 2007.

[5] H. A. Gil and G. Joos, "Customer-owned back-up generators for energy management by distribution utilities," IEEE Transactions on Power Systems, vol. 22, no. 3, pp. 1044-1050, 2007.

[6] M. E. Baran and I. M. El-Markabi, "A multiagent-based dispatching scheme for distributed generators for voltage support on distribution feeders," IEEE Transactions on Power Systems, vol. 22, no. 1, pp. 52 $59,2007$.

[7] H. A. Gil and G. Joos, "Models for quantifying the economic benefits of distributed generation," IEEE Transactions on Power Systems, vol. 23, no. 2, pp. 327-335, 2008.

[8] F. Aminifar and M. Fotuhi-Firuzabad, "Reliability-constrained unit commitment considering interruptible load participation," Iranian Journal of Electrical E Electronic Engineering, vol. 3, no. 1-2, pp. 10-20, 2007.

[9] T. S. Dillon, K. W. Edwin, H.-D. Kochs, and R. J. Taud, "Integer programming approach to the problem of optimal unit commitment with probabilistic reserve determination," IEEE Transactions on Apparatus and Systems, vol. 97, no. 6, pp. 2154-2166, 1978.

[10] J. D. Guy, "Security constrained unit commitment," IEEE Transactions on Power Apparatus and Systems, vol. 90, no. 3, pp. 1385-1390, 1971.

[11] F. Bouffard and F. D. Galiana, "An electricity market with a probabilistic spinning reserve criterion," IEEE Transactions on Power Systems, vol. 19, no. 1, pp. 300-307, 2004.

[12] H. B. Gooi, D. P. Mendes, K. R. W. Bell, and D. S. Kirschen, "Optimal scheduling of spinning reserve," IEEE Transactions on Power Systems, vol. 14, no. 4, pp. 1485-1492, 1999.

[13] S. H. Hosseini and E. Abbasi, "Probabilistic unit commitment with wind farms considerations," in Large Engineering Systems Conference on Power Engineering (LESCOPE '07), pp. 73-77, Montreal, Canada, 2007. 
[14] Wind Speed Data of Siahpoosh, MATN Research Center, Tehran, Iran.

[15] N. Alguacil and A. J. Conejo, "Multiperiod optimal power flow using benders decomposition," IEEE Transactions on Power Systems, vol. 15, no. 1, pp. 196-201, 2000.

[16] A. J. Wood and B. F. Wollenberg, Power Generation, Operation and Control, Wiley, New York, NY, USA, 1996.

[17] T. S. Dillon, K. W. Edwin, H. D. Kochs, and R. J. Taud, “Integer programming approach to the problem of optimal unit commitment with probabilistic reserve determination," IEEE Transactions on Power Apparatus and Systems, vol. 97, no. 6, pp. 2154-2166, 1978.

[18] M. Carrión and J. M. Arroyo, "A computationally efficient mixed-integer linear formulation for the thermal unit commitment problem," IEEE Transactions on Power Systems, vol. 21, no. 3, pp. 1371-1378, 2006.

[19] M. P. Nowak and W. Römisch, "Stochastic Lagrangian relaxation applied to power scheduling in a hydro-thermal system under uncertainty," Annals of Operations Research, vol. 100, no. 1-4, pp. 251-272, 2000.

[20] A. A. Chowdhury, "Reliability models for large wind farms in generation system planning," in IEEE Power Engineering Society General Meeting, pp. 1926-1933, June 2005.

[21] M. Shahidehpour, H. Yamin, and Z. Li, Market Operations in Electric Power Systems, John Wiley \& Sons, New York, NY, USA, 2002.

[22] J. F. Benders, "Partitioning procedures for solving mixed-variables programming problems," Numerische Mathematik, vol. 4, no. 1, pp. 238-252, 1962.

[23] N. I. Deeb and S. M. Shahidehpour, "Decomposition approach for minimising real power losses in power systems," IEE Proceedings C, vol. 138, no. 1, pp. 27-38, 1991.

[24] A. Zakariazadeh and S. Jadid, "Simultaneous clearing of wind-thermal generation and reserve market with interruptible load participation," International Review of Electrical Engineering, vol. 4, no. 6, pp. 1347-1356, 2009.

[25] M. E. Baran and F. F. Wu, "Network reconfiguration in distribution systems for loss reduction and load balancing," IEEE Transactions on Power Delivery, vol. 4, no. 2, pp. 1401-1407, 1992.

[26] F. Bouffard, F. D. Galiana, and A. J. Conejo, "Market-clearing with stochastic security-part I: formulation," in IEEE Power Engineering Society General Meeting (PES '06), p. 1, Montreal, Canada, June 2006. 


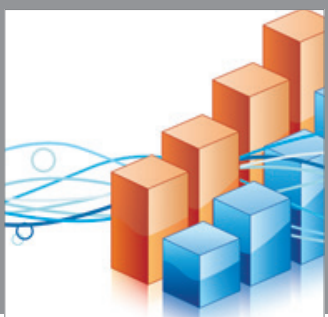

Advances in

Operations Research

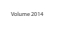

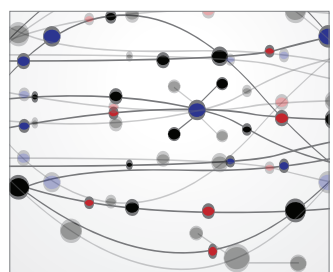

\section{The Scientific} World Journal
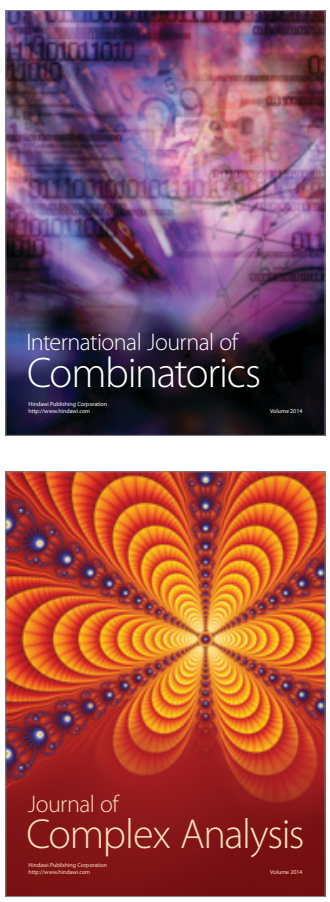

International Journal of

Mathematics and

Mathematical

Sciences
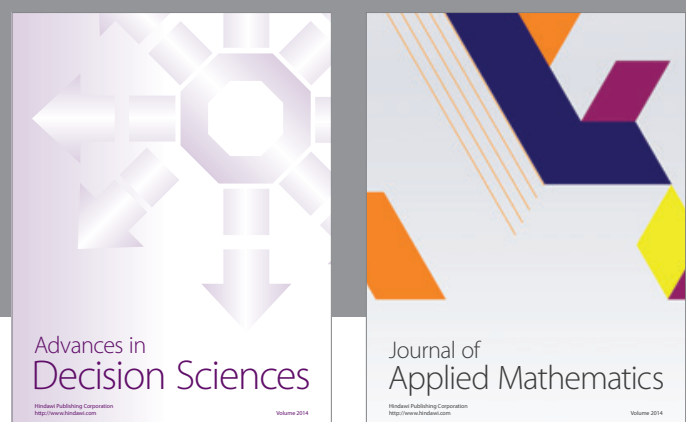

Journal of

Applied Mathematics
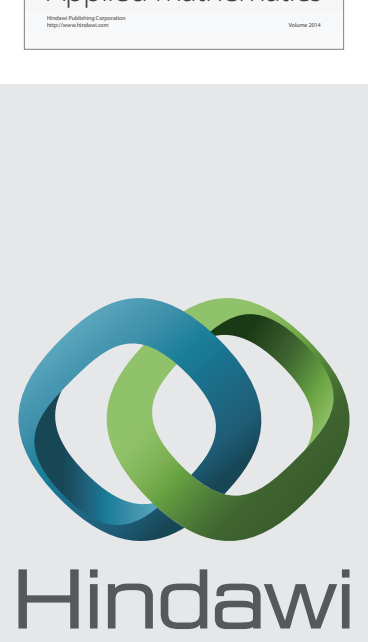

Submit your manuscripts at http://www.hindawi.com
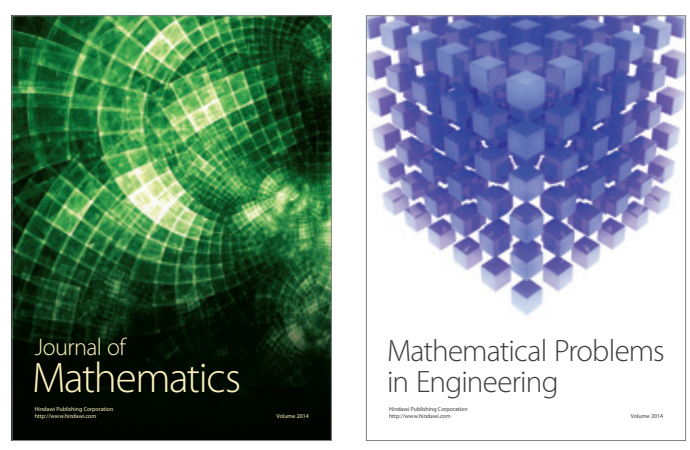

Mathematical Problems in Engineering
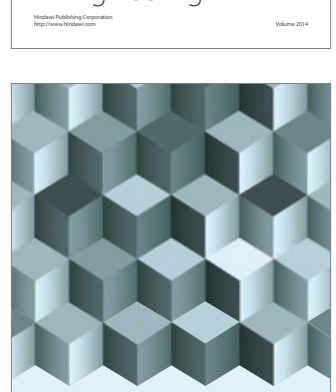

Journal of

Function Spaces
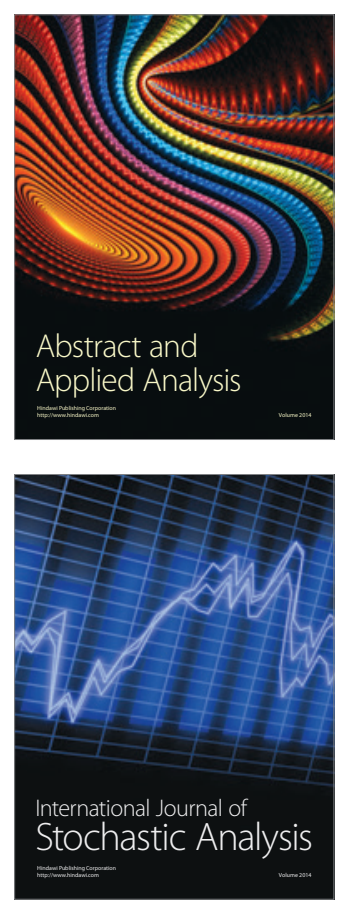

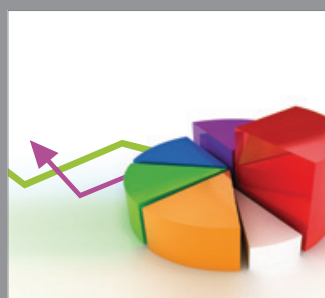

ournal of

Probability and Statistics

Promensencen
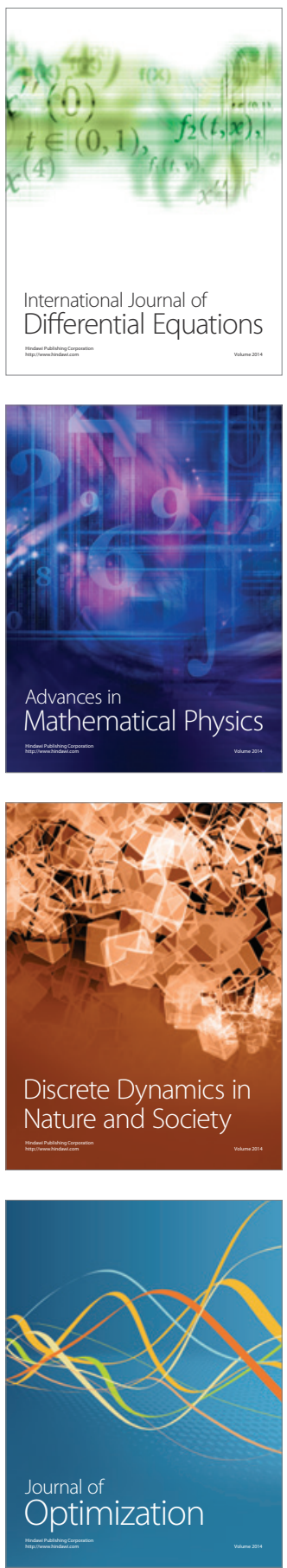